\title{
Laboratory activity for a new procedure of MIVIS calibration and relative validation with test data
}

\author{
Cristiana Bassani $\left({ }^{1}\right)$, Rosa Maria Cavalli $\left({ }^{2}\right)$, Angelo Palombo $\left({ }^{1}\right)$, \\ Stefano Pignatti $\left({ }^{1}\right)\left({ }^{2}\right)$ and Fabio Madonna $\left({ }^{1}\right)$ \\ (') Istituto di Metodologie per l'Analisi Ambientale (IMAA), CNR, Tito Scalo (PZ), Italy \\ $\left.{ }^{(}{ }^{2}\right)$ Laboratorio Aereo Ricerche Ambientali (LARA), IIA-CNR, Tor Vergata (RM), Italy
}

\begin{abstract}
Remotely sensed data, recorded by means of the MIVIS hyperspectral scanner in the framework of the research activity of the CNR Institutes IIA-LARA and IMAA, have been calibrated to reflectance values and then quantitatively compared with ground data. A new procedure for radiometric calibration has been defined by utilizing the MIVIS test-bench and applying a wider radiance range with respect to the one provided by the manufacturing company. New calibration curves have been determined and applied in the pre-processing chain. For validation purpose ground spectra were measured during the campaign by means of a portable spectroradiometer. The atmospheric correction has been carried out by implementing an IDL procedure to manage MODTRAN4 input and output cards. MIVIS test data acquired over Passo Corese (Roma) have shown how the new calibration coefficients significantly improve the radiometric accuracy. In particular, in the VIS spectral region the percentage error, with respect to a ground truth spectrum, is about half of that occurring if the standard calibration coefficients are used.
\end{abstract}

Key words radiometric calibration - MIVIS - atmospheric correction - MODTRAN

\section{Introduction}

A quality index of radiometrically calibrated and atmospherically corrected data can be obtained by comparing spectral ground truths acquired at the same time of flight (Clark et al., 2002).

This ground truths area must be characterized by a good spectral and spatial homogeneity and a

Mailing address: Dr. Cristiana Bassani, Istituto di Metodologie per l'Analisi Ambientale (IMAA), CNR, C.da S. Loja, 85050 Tito Scalo (PZ), Italy; e-mail: bassani@imaa.cnr.it high reflectance to assure a high signal perceived at the sensor. Such comparison between spectra measured by the sensor and ground truths has been applied to data collected during last MIVIS (Multispectral Infrared and Visibile Imaging Spectrometer, AA500, Daedalus Inc.) campaigns performed by CNR. MIVIS is a whiskbroom scanner in which the light collimated by the scan head is distributed by means of dichroic filters into 4 spectrometers that simultaneously record the radiation coming from the Earth (table I). The scanner geometry is characterized by a FoV of $71.06^{\circ}$, an IFoV $2 \mathrm{mrad}$ and a scan rotation frequency varying from $6.25 \mathrm{~Hz}$ to $25 \mathrm{~Hz}$; data are digitized at 12 bit (Daedalus, 1993).

MIVIS standard radiometric calibration sequence is carried out on a Test Bench (TB) made up of a black painted aluminium box with two calibration halogen lamps and a reference panel of barium sulphate (Spectraflect, reflectance cer- 
tified in 1994) of known reflectance. The MIVIS sensor is mounted on the bench in the lab for the automatic Acceptance Test Procedure (ATP), which is run for a fast and periodic scanner calibration and a performance check (signal to noise ration - SNR in fig. 1).

Such standard calibration associates the $\mathrm{DN}$, measured during the test, to the panel radiance values tabulated for each channel, so producing the calibration curves, straight lines intersecting the axis at the origin, relating DN with tabulated radiance values. The straight line slope determines the Radiance Factor (RF) applied to the radiometric calibration to convert the $\mathrm{DN}$ to radiance while the standard deviation of the DN values has been considered an estimation of the SNR.

Table I. MIVIS spectral characteristics.

\begin{tabular}{cccc}
\hline \hline Spectrometer & $\begin{array}{c}\text { Bands } \\
(\mathrm{nm})\end{array}$ & $\begin{array}{c}\text { Spectral range } \\
(\mathrm{nm})\end{array}$ & Bandwidth \\
\hline 1 & $1-20$ & $430-830$ & 20 \\
2 & $21-28$ & $1150-1550$ & 50 \\
3 & $29-92$ & $1983-2478$ & 9 \\
4 & $93-102$ & $8180-12700$ & $340 \div 440$ \\
\hline
\end{tabular}

From 1994 to date, besides the common checks performed at the Deadalus labs, a trial (Lechi, 2000) of reliability of the RF values defined by ATP has been carried out as regards possible variations of the TB parameters (lamps and panels). Lechi's work is not to be considered resolutive, but it certainly represents an outstanding experimental basis to define a more precise procedure.

The present work describes a non standard procedure, implemented in the lab using the TB and a reference spectroradiometer to obtain new RF curves on the basis of a wider range of lighting conditions to better describe the natural irradiance values. The new calibration coefficients have been applied to the test image recorded on an cultivated area close to Passo Corese (Rome, Italy) on March 7, 2002 at an altitude of $1500 \mathrm{~m}$ a.s.l (pixel ground resolution 3 $\mathrm{m} /$ pixel) where spectral ground truth campaign has been performed for image validation purpose.

Furthermore, the paper includes an Appendix to demonstrate analytically and experimentally how the optical defocusing occurring in the TB during ATP does not invalidate the accuracy of the MIVIS calibration. Thus, the presence of a collimator facing the scan head is not necessary for a correct calibration.

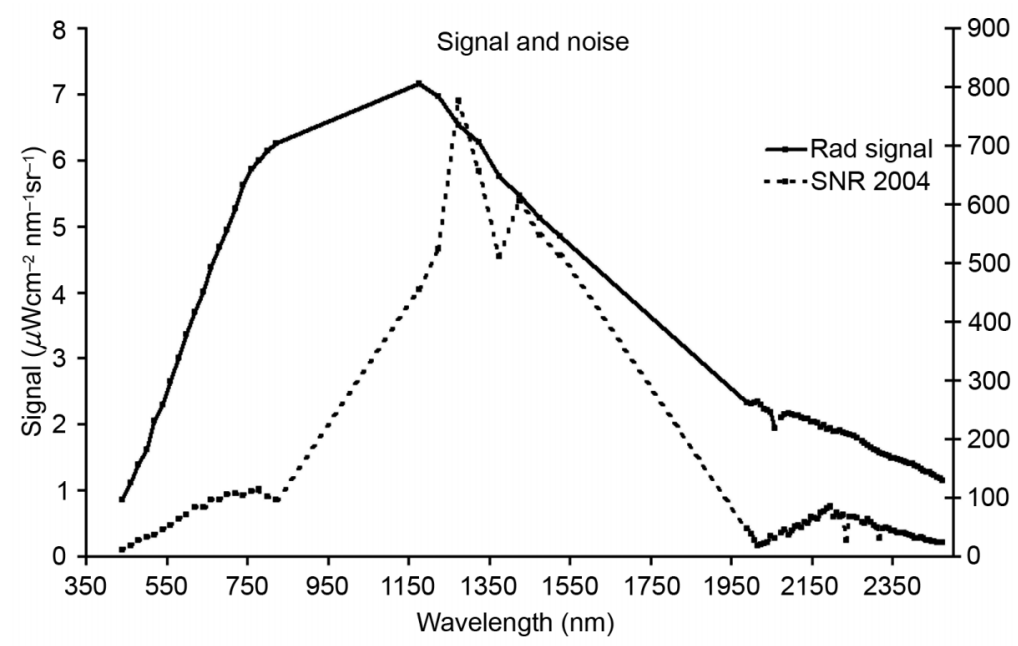

Fig. 1. MIVIS SNR @ $25 \mathrm{~Hz}$, plus the radiance values from which it has been calculated. 


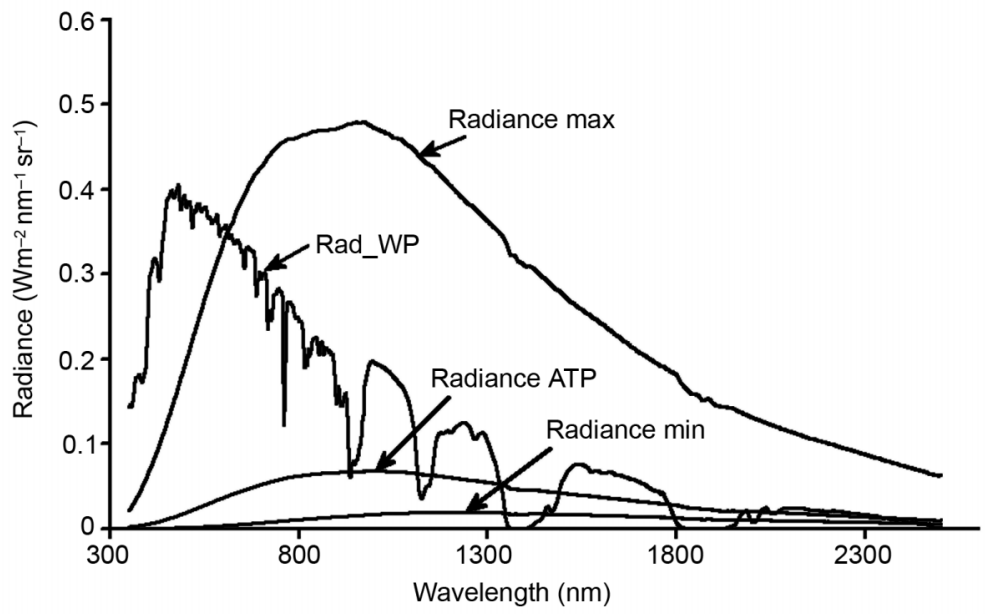

Fig. 2. Radiances inherent to the radiometric calibration process: Rad_WP: radiance of Spectralon panel measured in open air at our latitude in summer time; Rad_max (maximum radiance) and Rad_min (lowest radiance) measured in TB in the new procedure; Rad_ATP: radiance in the TB during ATP.

\section{MIVIS radiometric calibration}

In the ATP the method used to calculate the panel radiance data for the $\mathrm{TB}$ is based on the following equation:

$$
N_{\lambda}=2 \cdot\left[\left(\frac{H_{\lambda} \sin \varphi}{\pi}\right) \cdot e_{\lambda}\right]
$$

where $N_{\lambda}$ is the panel radiance at wavelength $\lambda$, $H_{\lambda}$ the panel irradiance at wavelength $\lambda ; \varphi$ the lamp to panel illumination angle; $e_{\lambda}$ the panel reflectance at wavelength $\lambda$, and $\pi$ the factor for Lambert surface. The physical quantities involved in eq. (2.1) are all monochromatic and the $\lambda$ index corresponds to the central wavelength of each channel of the sensor.

In this procedure the Radiance Factors for the first 92 channels are derived from this simple relation

$$
K_{\lambda}=\frac{\mathrm{DN}_{\lambda}-\mathrm{DN}_{\lambda \mathrm{REF} 1}}{N_{\lambda}}
$$

where $\mathrm{DN}_{\lambda \mathrm{REF} 1}$ is the offset of dark current (DN of the first MIVIS internal blackbodies).

During the analysis it was noted that the radiance received by the instrument during the ATP is much lower than the one measured throughout instrument's normal operation con- dition (summer clear sky) (fig. 2). Therefore, to verify the behavior of the scanner operating under radiance conditions similar to those occurring during surveys, a new calibration procedure was tuned.

The developed calibration procedure consists in building calibration curves with the use of different radiance values obtained by either varying the power of the lighting source (introducing additional lamps besides those normally used for ATP) or employing different reflectance panels. In the experimental phase the combination of different irradiating powers and different panels produced 18 experimental conditions measured by both MIVIS and the portable spectrometer.

The panels employed during non standard calibration are: the Spectraflect (BaSO4) provided with the TB; the Spectralon certified by LabSphere Inc. up to December $2002(\Delta \rho \leq$ $\leq \pm 0.005$ between $300-2200 \mathrm{~nm}$ and $\Delta \rho \leq$ $\leq \pm 0.020$ between $2250-2500 \mathrm{~nm}$ ); a $18 \%$ Kodak grey panel and the TB black panel. They have been measured by means of the portable ASD FieldSpec Pro FR spectroradiometer (ASD). ASD is a portable spectrometer covering the range between $350 \mathrm{~nm}$ and $2500 \mathrm{~nm}$ with a spectral resolution of $3 \mathrm{~nm}$ for the range $350-1000$ 
$\mathrm{nm}$, and of $10 \mathrm{~nm}$ for the 1000-2500 nm (Milton et al., 1997). In accordance with the last calibration certificate the error associated with the radiance measures is: $1.4 \times 10^{-9} \mathrm{~W} / \mathrm{cm}^{2} \mathrm{~nm} \mathrm{sr} @$

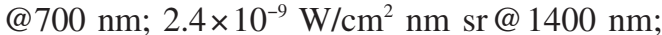
and of $10.4 \times 10^{-9} \mathrm{~W} / \mathrm{cm}^{2} \mathrm{~nm} \mathrm{sr} @ 2100 \mathrm{~nm}$.

The $\mathrm{DN}_{\lambda}$ used to express the function relationship with the radiance are not those utilized by the TB software performing the ATP, but they were obtained off-line by reading the DN directly from the raw files recorded on the MIVIS data tape. Each raw file recorded for each panel differently illuminated has a length

Channel 21

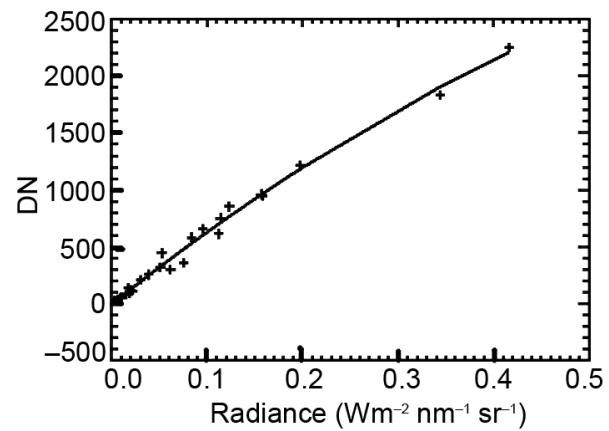

of about 400 scan lines at a scan rotation frequency of $25 \mathrm{~Hz}$.

The $\mathrm{DN}_{\lambda}$ used in the RF calculation are the mean computed on a $20 \times 20$ pixel square, selected in the middle of the scanner scan line, once the dark currents are subtracted. This window size was chosen to avoid image blurring due to the reduced distance between the panel and scan head, which does not permit panel images to be well focused, and to allow a significant statistical assessment. The square standard deviation takes into consideration both measurement variations typical of the scanner, which is a least

Fig. 3. Best fit of the 18 radiance values for channel 21 (second polynomial fit) and channel 31 (linear fit) to derive the calibration curves.
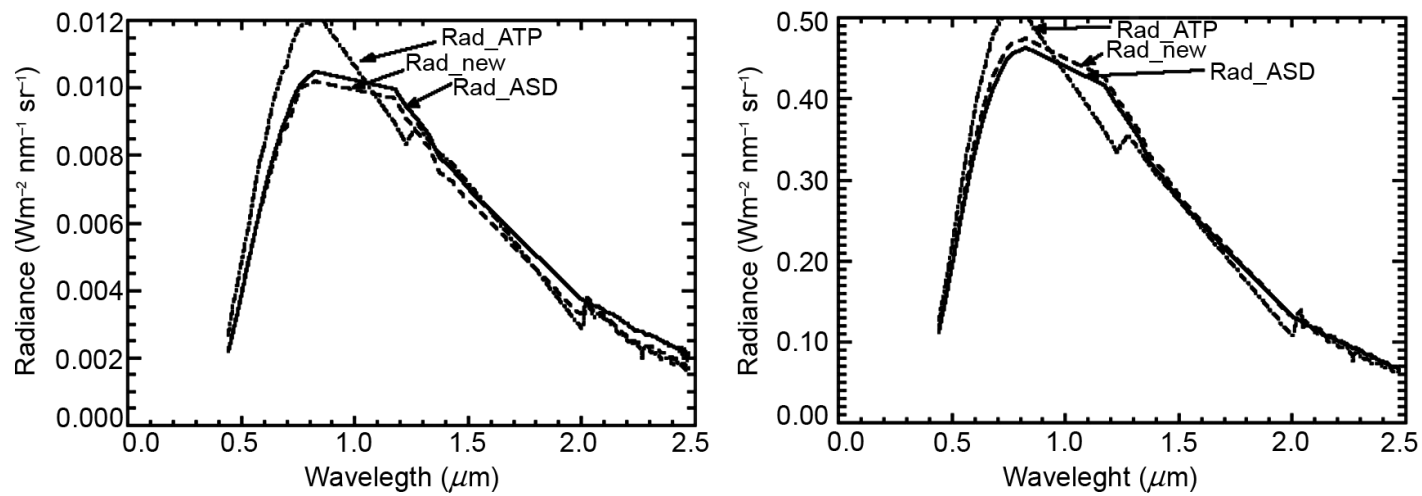

Fig. 4. Comparison of Spectralon radiance measured in the TB. Rad_ATP curve is the MIVIS radiance calibrated with RF from ATP; Rad_new is the MIVIS radiance calibrated with the new procedure; Rad_ASD is the ASD measured inside the TB. 
contribution, and the possible error due to the non homogeneities in the panel lighting.

The 18 couples of values (Radiance/DN) so defined for each channel, positioned on the graph of fig. 3, show that the linear trend, presumed as valid by the TB software during the ATP, is not always perfectly verified. For example, some points of the channel 21 (fig. 3 on the left) wander off from the hypothesized linear trend at higher radiance values (close to those occurring under MIVIS normal working conditions).

In the first 28 channels (first and second spectrometer, VIS-NIR regions) the best fitting curve is a second polynomial, while for the MIR the trend is a perfect straight line (fig. 4 on the right). To determine the RF of the non linear channels, instead of a linear relationship, the following equation is used

$$
\mathrm{DN}=a \cdot \mathrm{RAD}^{2}+b \cdot \mathrm{RAD}+c
$$

where $a, b$ and $c$ are the fit coefficients (Bassani et al., 2002).

To ascertain the accuracy of the MIVIS radiance values obtained by applying the new calibration they were compared with the radiance values obtained by applying the RF from the ATP, and the measured panel radiance by ASD. Figure 4 depicts how the panel radiance curves, obtained with the new calibration coefficients, better fits the reference ASD curve to those calculated by using the ATP. The difference between the ATP and ASD curves could be primarily due to variations of the TB lamp irradiance and the weathering conditions of the Spectraflect panel (Rollin, 2000). As a consequence, MIVIS radiometric correction was performed using the new calibration coefficients.

\section{MIVIS atmospheric correction and validation}

To convert the instrument perceived radiance values to reflectance $\rho_{g}(\lambda)$, an intrinsic property of the surface independent of the illumination and atmospheric conditions at the time of measurements, an atmospheric correction procedure was applied to the MIVIS data. In order to cor- rect the atmospheric effects, the relationship between the upward radiance, $L_{0}(\lambda)$, measured by MIVIS and the surface reflectance, $\rho_{g}(\lambda)$, has to be established by using the MODTRAN4 radiative transfer model (Slater, 1980; Tanré, 1992; Vermote et al., 1997).

Under the assumption of a cloudless sky, a plane-parallel atmosphere and a lambertian surface, the general model applied is given by

$$
\rho_{g}(\lambda)=\frac{\pi\left[L_{0}(\lambda)-L_{d \uparrow}(\lambda)\right]}{\tau_{v \uparrow}(\lambda)\left[E_{0}(\lambda) \cos \theta_{z} \tau_{z \downarrow}(\lambda)+E_{d \downarrow}(\lambda)\right]}
$$

where $L_{0}(\lambda)$ is the radiance measured at the sensor; $L_{d \uparrow}(\lambda)$ represents the upwelling atmosphere spectral radiance, scattered from the direct sunbeam into the sensor by the atmosphere (path radiance); $E_{d \downarrow}(\lambda)$ is the downwelling spectral irradiance at the surface due to the scattered solar flux in the atmosphere; $\tau_{v \uparrow}(\lambda)$ is the atmospheric transmittance along the path from the ground surface to the sensor; $\tau_{z \downarrow}(\lambda)$ is the atmospheric transmittance along the path from the sun to the ground surface; $\theta_{z}$ is the solar zenith angle, $E_{0 \downarrow}(\lambda)$ is the solar spectral irradiance at the top of the Earth's atmosphere.

For applying the atmospheric correction to MIVIS data, an IDL procedure has been developed to manage input and output cards, to run MODTRAN4 (Berk et al., 1999). The IDL code is a stepwise procedure in which the different atmospheric parameters are computed separately. The first step takes into consideration the contribution of path radiance, $L_{d \uparrow}(\lambda)$ along the scan line, while in the following step the quantities $L_{d \uparrow}(\lambda), E_{d \downarrow}(\lambda), \tau_{v \uparrow}(\lambda), \tau_{z \downarrow}(\lambda), E_{0 \downarrow}(\lambda)$ are computed. The procedure automatically resamples each MODTRAN output quantity $F_{i}(\lambda)$ to MIVIS spectral channels by using the following formula:

$$
F_{j}(\lambda)=\frac{\sum_{i=1}^{n} F_{i}(\lambda) \cdot\left[r_{i}(\lambda)\right]_{j}}{\sum_{i=1}^{n}\left[r_{i}(\lambda)\right]_{j}}
$$

where the index $i$ is the wavelength step selected in the MODTRAN simulations ( $1 \mathrm{~nm}$ in our case), $j$ is the MIVIS channels index (i.e. 1-92), 
and $\left[r_{i}(\lambda)\right]_{j}$ is the spectral response function for the $j$ MIVIS channel in the MODTRAN wavelength step. The determined $F_{j}(\lambda)$ are applied in eq. (3.1) to obtain the MIVIS reflectance value.

The IDL code was applied to correct the MIVIS survey on Passo Corese configuring MODTRAN cards with a mid-latitude summer profile and with a rural aerosol model (visibility equal to $23 \mathrm{~km}$ ).

The validation process of the atmospheric correction is based on the comparison of the
MIVIS reflectance with the spectral ground reflectance spectra acquired with the ASD. To fulfil this task the selection of a wide Areas Of Interest (AOI), as spectrally and spatially homogeneous as possible, is required, so to minimize the adjacency effects; moreover high reflectance values are required in order to have high SNR at the sensor.

As regards the image of Passo Corese, the cultivated fields were not used as target reference (mixture of soil and bean plants) because of the

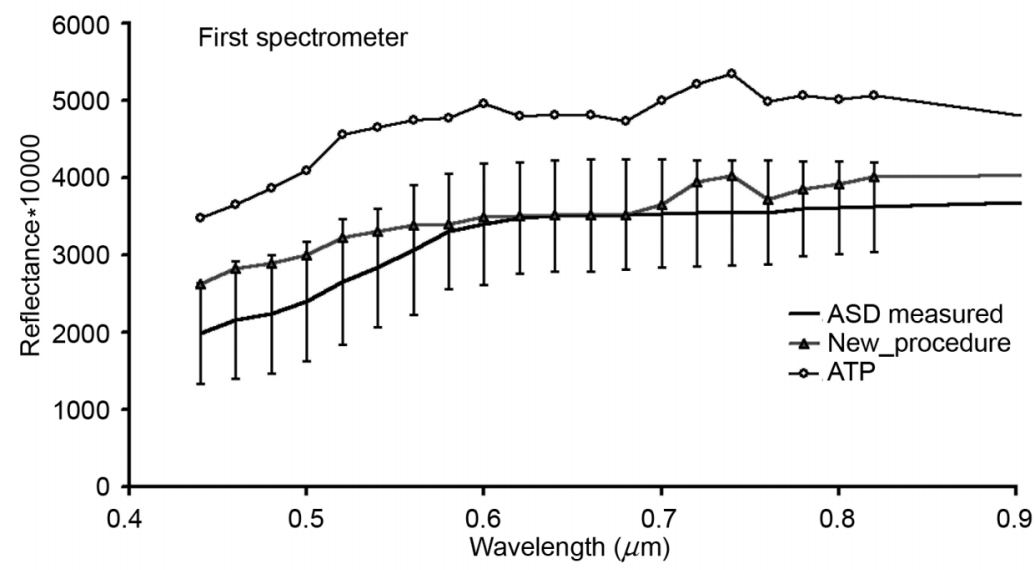

Fig. 5. Comparison of APT and new calibration spectrum of the AOI in concrete with respect to ASD reflectance. ASD measure is graphed with one sigma errors bars.

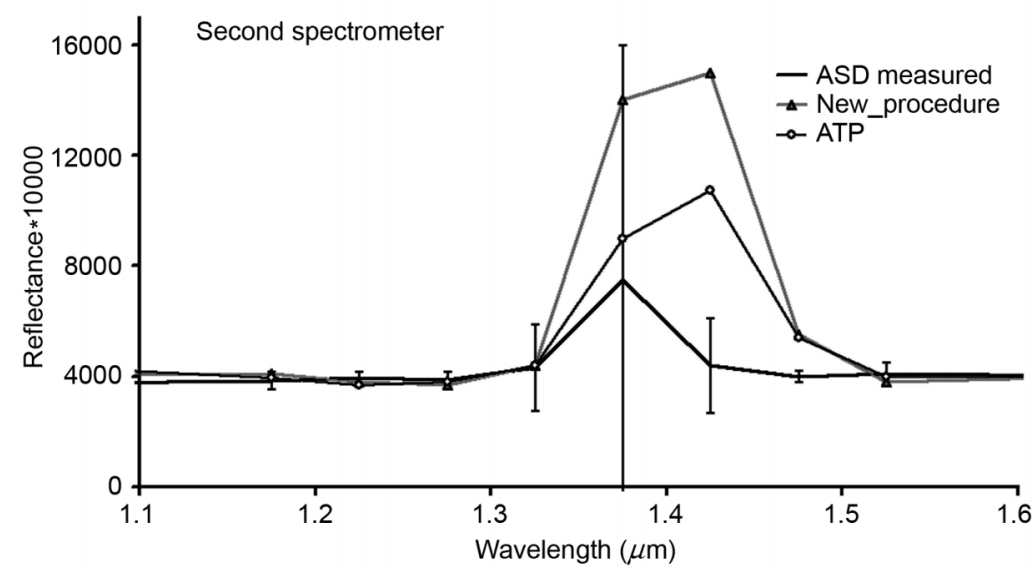

Fig. 6. Comparison of APT and new calibration spectrum of the AOI in concrete with respect to ASD reflectance. ASD measure is plotted with one sigma errors bars. 


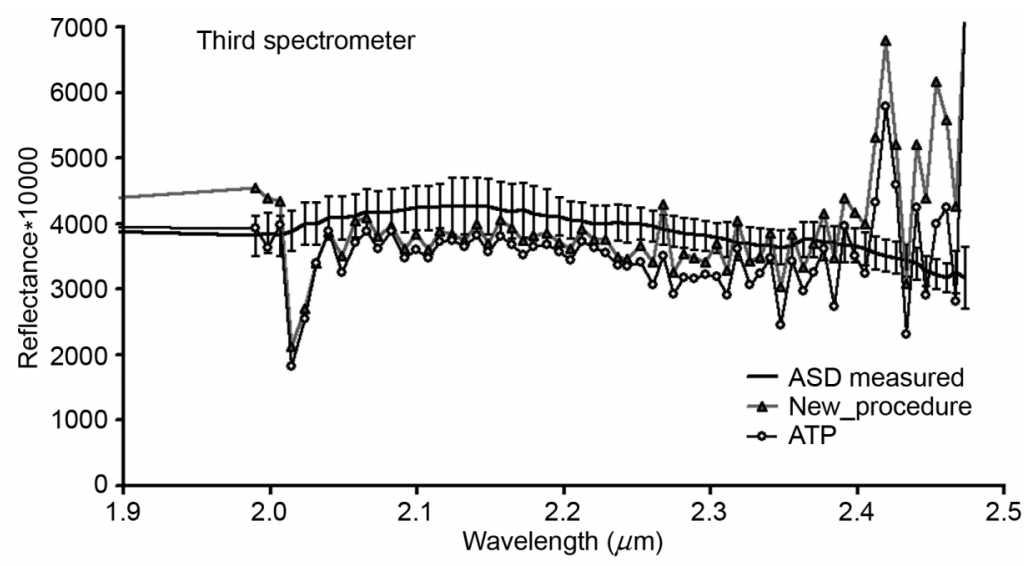

Fig. 7. Comparison of APT and new calibration spectrum of the AOI in concrete with respect to ASD reflectance. ASD measure is plotted with one sigma errors bars.

growing season and, therefore, the AOI were selected on a concrete platform covering a total area of about $200 \mathrm{~m}^{2}$. The following graphs in figs. 5, 6 and 7 show the MIVIS reflectance spectra obtained respectively by applying the radiometric calibration with the RF derived from the ATP and the new calibration coefficients from the above described new procedure. On the graphs MIVIS spectra are compared with the ASD measurements of the AOI. ASD measurements have been averaged and the standard deviation has been considered like the spectral variability of the concrete spectrum (error bars on the graphs).

The comparison of MIVIS and ground spectra outlined how the ATP tends to overestimate the reflectance values in the VIS region (fig. 5) where the reflectance value determined by means of the new procedures is to be considered equal within the error to the mean of the ground truths. In terms of percentage with the new calibration the reflectance errors are of about $15 \%$ with respect to the ASD ground truth, while the ATP carries an error of about $30 \%$.

The NIR region is manifestly subject to a contribution of water vapour (fig. 6) as regards the ground truths as well. The high variability of the water vapour content in the ground spectra leads to a great uncertainty in determining the reference reflectance value so much that both MIVIS spectra fall within the ASD meas- urements confidence interval. In the SWIR region (fig. 7), the two calibration procedures instead show comparable reflectance values, even though both highlight an underestimation of the image reflectance with respect to the reference ground truth spectra.

\section{Results and discussion}

In order to test the accuracy of the RF for the entire instrument dynamic range, a new procedure has been devised. Such non-standard procedure is able to produce radiance values with a radiometric precision higher than that reachable by the ATP procedure. The non-standard procedure, being developed not taking into account the ATP tabulated values, decouples the scanner performance variation from that related to the TB.

By comparing the new procedure with the ATP in the data pre-processing chain to obtain reflectance values, it has been observed that the ATP in the VIS region overestimates the spectrum of the selected AOI. This result is congruent with the experimental results obtained from the calibration of the TB DN 18 images, showing the overestimation of the panels' radiance in the ATP. In the NIR and SWIR spectral regions, the ATP and the new calibration procedure attain two similar curves both occurring nevertheless in the sec- 
ond spectrometer within the intrinsic AOI variability, while in the third MIVIS optical port they both appear below the ASD reflectance curve.

The result is satisfactory if compared to the purpose of this work: the new procedure brings about an indeterminacy in the reflectance measurement which is about the half of that occurring if the ATP RF are used.

Once the experimental phase is over, this procedure can be applied at regular intervals throughout the year to verify, and possibly update, the ATP routine calibration.

The new radiometric calibration is going to be applied in the next MIVIS airborne campaign to be deployed in Sweden in June 2003, where atmospheric correction will be performed using in situ atmospheric measures (i.e. radiosounding, solar direct measurements for optical thickness), thus permitting the uncertainty of MODTRAN simulations to be reduced.

\section{Acknowledgements}

The authors are grateful to Maurizio Poscolieri and Roberto Ponzianelli for valuable discussions and to Angela Mirabelli for helping to improve the paper.

Appendix. ATP accuracy as function of the optical defocusing within the TB.

To demonstrate how the distance between the TB panel and the MIVIS scan head is not affecting the calibration procedure, a twofold procedure has been followed: the former, analytic, according to the rules of geometric optics; the latter, experimental, to prove the independence of the radiance measures on the distance from a uniform and wide source.

A telescope could be schematised with a thin lens (fig. A.1), in which, for distances smaller than the infinite, the image will be displayed at a distance $I$. The difference between $I$ and the focal distance $(F)$ is named defocalization $(D)$. The distance $I$, for a point $C$ located at $Z$ distance from the lens, is defined by the following equation (formula valid for thin lens)

$$
I=\frac{Z F}{Z-F} .
$$

Therefore, the defocalization is defined as

$$
D=\frac{F^{2}}{Z-F} .
$$

From the above equations, it derives that the defocalization goes asymptotically to zero by increasing the distance. The defocusing phenomenon causes a lost of energy passing the field stop, coming from the single point of the viewed surface, that is due to the non coincidence of the image disc and the field stop located on the focal plane (fig. A.1- $\beta$ ). Nevertheless, it is possible to demonstrate that: from a surface, sufficiently large and uniformly illuminated, the energy reaching the sensor after the field stop is not correlated to the distance.

A generic element of the surface $C$ at distance $Z$ from the lens, forming an angle $\theta$ with the optical axis, is focalised at position $C^{\prime}$ and it is spread on the focal plane $F$, as a circle (disc image) of diameter $E H$. The diameter of the above mentioned circle $\left(2 R_{c}\right)$ is yielded by

$$
E H=E G+G H .
$$

By means of a geometric consideration

$$
\frac{E G}{D}=\frac{R_{t}+O C^{\prime}}{I} \quad \frac{G H}{D}=\frac{R_{t}-O C^{\prime}}{I}
$$




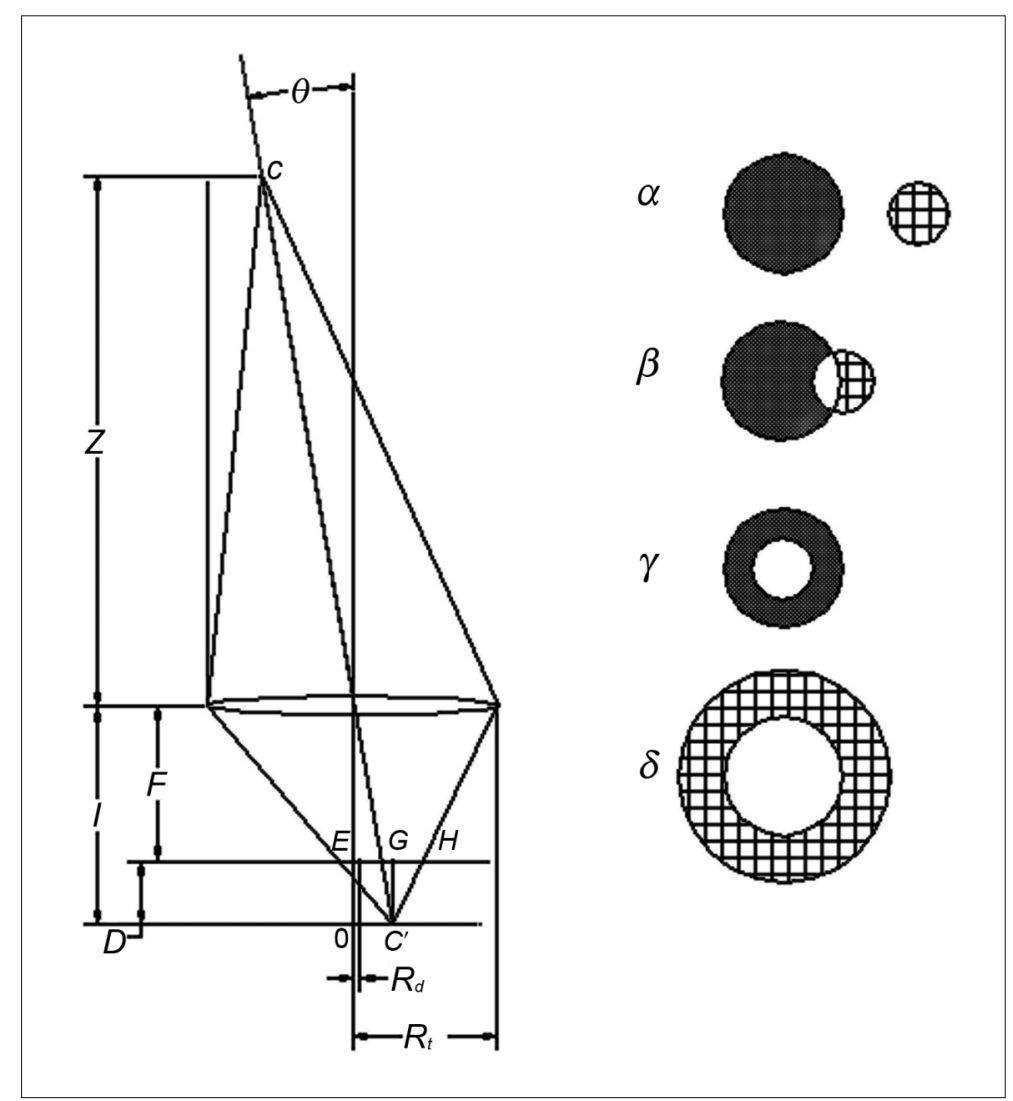

Fig. A.1. Telescope scheme with one convergent lens. On the right $(\alpha)$ is the front view of the field stop (grey disc) and the image of $C$ on the focal plane (light disc patterned with squares); $(\beta)$ show the relative position and dimension of the discs for condition $\tau_{1} ;(\gamma)$ show the relative position and dimension of the discs for $\tau_{3} ;(\delta)$ show the relative position and dimension of the discs for $\tau_{2}$. On $\beta, \gamma, \delta$ white area point out the two discs intersection.

Where $R_{t}$ is the lens radius and $O C^{\prime}$ is the parallax $(P)$ which is determined by the following relation:

$$
P=I \tan (\vartheta)
$$

Including eq. (A.4) in eq. (A.3), the new relationship is obtained

$$
E H=D \frac{R_{t}+O C^{\prime}}{I}+D \frac{R_{t}-O C^{\prime}}{I} .
$$

From which it derives that

$$
\frac{E H}{2}=D \frac{R_{t}}{I} \quad \frac{E H}{2}=\frac{F R_{t}}{Z} .
$$

Assuming $E_{0}$ as the energy falling on the telescope from the surface viewed under the infinitesimal solid angle $\delta \omega$, it is spread on the focal plane within a circle of radius $R_{c}$. The fraction of $E_{0}$ passing 
the field stop is described by the $\tau$ function. The $\tau$ function is defined according to three different formulas (fig. A.1).

In the condition of partial overlap between field stop and disc image $\tau_{1}$ (fig. A.1- $\beta$ )

$$
\tau_{1}=\frac{A}{\pi R_{c}^{2}}
$$

where $A$ represents the overlap area between disc image and field stop and is yielded by the relation

$$
\begin{aligned}
& A=R_{c}^{2} \arccos \left(\frac{P^{2}+R_{c}^{2}-R_{d}^{2}}{2 d R_{c}}\right)+R_{d}^{2} \arccos \left(\frac{P^{2}-R_{c}^{2}+R_{d}^{2}}{2 d R_{d}}\right)+ \\
& -\frac{1}{2} \sqrt{\left(-P+R_{c}+R_{d}\right)\left(P+R_{c}-R_{d}\right)\left(P-R_{c}+R_{d}\right)\left(P+R_{c}+R_{d}\right)}
\end{aligned}
$$

where $P$ is the parallax and corresponds to the distance between the centres of field stop and disc image.

In the condition of total overlap between field stop and disc image and in the case of $R_{c} \geq R_{d}$ (fig. A. $1-\gamma)$ the $\tau$ function is expressed by

$$
\tau_{2}=\frac{R_{d}^{2}}{R_{c}^{2}}
$$

In the situation of total overlap between field stop and disc image with $R_{c} \leq R_{d}$ (fig. A.1- $\delta$ ) the $\tau$ function is expressed by

$$
\tau_{3}=1
$$

The condition of $R_{c}=R_{d}$ implies that $Z=F R_{t} / R_{d}$ and, therefore, for higher $Z$ values the disc image has dimensions lower than the field stop.

The energy fraction $\hat{\rho}$, arriving to the sensor from the whole surface located at distance $Z$, is obtained by integrating the $\tau$ function for the entire solid angle, lying within the sensor viewing angle. For $Z \leq F R_{t} / R_{d}$ energy fraction $\hat{\rho}$ is obtained by

$$
\widehat{\rho}=2 \pi \int_{0}^{\theta_{\max }^{m_{2}}} \tau_{2} \operatorname{sen} \theta d \theta+2 \pi \int_{\theta_{\max }}^{\theta_{\max }^{m_{2}}} \tau_{1} \operatorname{sen} \theta d \theta
$$

while for $Z>F R_{t} / R_{d}$ by

$$
\widehat{\rho}=2 \pi \int_{0}^{\theta_{\max }^{\prime}} \tau_{3} \operatorname{sen} \theta d \theta+2 \pi \int_{\theta_{\max }}^{\theta_{\max }^{\mathrm{m}}} \tau_{1} \operatorname{sen} \theta d \theta .
$$

The integration boundaries $\theta_{\max }^{\prime}$ and $\theta_{\text {max }}^{\prime \prime}$ are the limit angles beyond which the disc image on the focal plane: 1$)$ does not totally overlap the field stop $\left.\left(\theta_{\max }^{\prime}\right) ; 2\right)$ is totally out of the field stop aperture $\left(\theta_{\max }^{\prime \prime}\right)$. The geometric condition that identifies $\theta_{\max }^{\prime}$ and $\theta_{\max }^{\prime \prime}$ are respectively

$$
\begin{aligned}
& E G-O C^{\prime}=0 \\
& E G-O C^{\prime}=-R_{d} .
\end{aligned}
$$

Utilizing eqs. ((A.1), (A.2)) and the first expression of eq. (A.4) and considering that $O C^{\prime}=P=I \tan (\theta)$, the next relation is derived

$$
\theta_{\text {max }}^{\prime}=\arctan \left(\frac{R_{t}}{Z}\right) \quad \theta_{\text {max }}^{\prime \prime}=\arctan \left(\frac{R_{t}}{Z}+\frac{R_{d}}{F}\right) .
$$




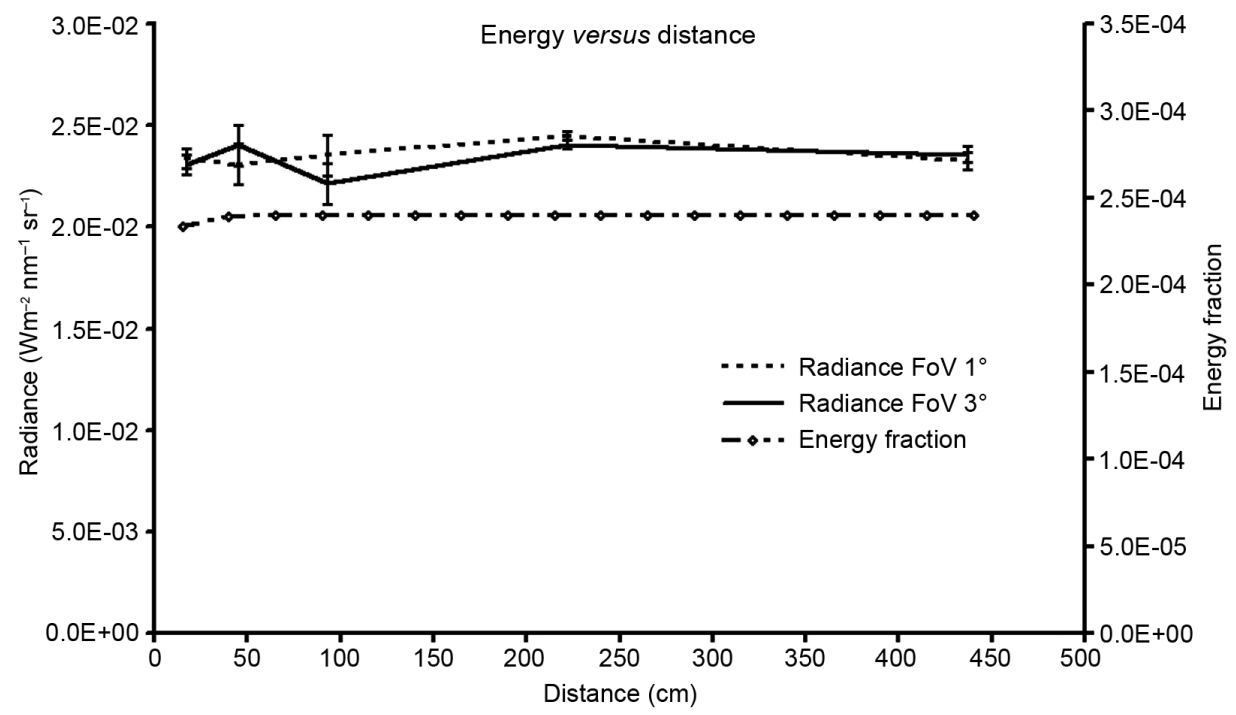

Fig. A.2. Graph of the radiance trend with respect to the distance from the panel to ASD foreoptics. The curves show the ASD measured trend for FoV $1^{\circ}(F=98 \mathrm{~mm})$ and FoV $3^{\circ}(F=32 \mathrm{~mm})$, while energy fraction curve represents the numerical solution of eq. (A.12) and eq. (A.13).

Table A.I. $R_{c}$ as a function of the distance between field stop source for the two ASD optics.

\begin{tabular}{ccc}
\hline \hline$Z(\mathrm{~cm})$ & $R_{c} 98(\mathrm{~mm})$ & $R_{c} 32(\mathrm{~mm})$ \\
\hline 17.5 & 14.00 & 1.65 \\
45.0 & 5.44 & 0.64 \\
93.0 & 2.63 & 0.31 \\
222.0 & 1.10 & 0.13 \\
437.0 & 0.56 & 0.07 \\
\hline
\end{tabular}

Numerically resolving the integral of eq. (A.12) and eq. (A.13), the trend of $\hat{\rho}$ energy fraction as function of the distance $Z$ is obtained. This $\hat{\rho}$ trend, only for distances very close to $F$, shows a difference from the asymptotic value. The difference is related to the methods applied to resolve the integral; in fact it decreases with the increase of the steps number used in the integration increase. At the distance in the TB used for the MIVIS calibration $\cong 70 \mathrm{~cm}$ (distance panel - scan head $\cong 40 \mathrm{~cm}$ plus the optical path within the MIVIS from the scan head to primary mirror) the error is lower of $0.2 \%$ (fig. A.2).

To obtain an experimental verification, an apparatus composed by a Spectralon panel illuminated by two halogen lamps, and the ASD has been arranged. The ASD has been used with two foreoptics (focal distance respectively of $98 \mathrm{~mm}$ and $32 \mathrm{~mm}$ with radius $R_{t}$ of $25 \mathrm{~mm}$ and $9 \mathrm{~mm}$ ) mounted on the fiber optic which operate as field stop with a diameter of $1.7 \mathrm{~mm}$.

Radiance measurements have been performed varying the foreoptics and panel distance. In table A.I the $R_{c}$ values, calculated for both foreoptics at the various distances, are shown. The $R_{c}$ values are ten times the field stop $\left(R_{d}\right)$ dimension. The graph of radiance @ $750 \mathrm{~nm}$ (fig. A.2) depicts the non- 
functional dependence on the radiance from the distance and the foreoptic field of view. Also in the

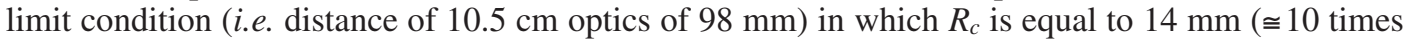
$R_{d}$ ) the radiance ASD measure is basically the same as those measured in the other experimental configurations $\left(R_{d}>>R_{c}\right)$.

Therefore, both analytically and experimentally demonstrations show how the optical defocusing, occurring in the TB during ATP, does not invalidate the accuracy of the MIVIS calibration.

\section{REFERENCES}

Bassani, C., R.M. Cavalli, A. Palombo and S. Pignatti (2002): La calibrazione dello scanner MIVIS (Multispectral Imaging Visibile and Infrared Spectrometer) nelle campagne di misura realizzate dal CNR nel 2001-2002, in Atti VI Conferenza Nazionale ASITA «Geomatica per l'Ambiente, il Territorio e il Patrimonio culturale», vol. I, p. 337.

Berk, A., G.P. Anderson, L.S. Bernstein, P.K. Acharya, H. Dothe, M.W. MatThew, S.M. AdleR-Golden, J.H. Chetwynd JR., S.C. Richtsmeier, B. Pukall, C.L. Allred, L.S. JeOng and M.L. HoKe (1999): MODTRAN4 radiative transfer modeling for atmospheric correction, in Optical Spectroscopic Techniques and Instrumentation for Atmospheric and Space Research III, SPIE Proc., 3756, 348-353.

Clark, R.N., G.A. Swayze, K.E. Livio, R.F. Kokaly, T.VV. King, J.B. Dal ton, J.S. Vance, BW. Rocwell, T. Hoefen and R.R. McDougal (2002): Surface reflectance calibration of terrestrial imaging spectroscopy data: a tutorial using AVIRIS, in Proceedings of the AVIRIS Conference, Pasadena, JPL, NASA.

DAedAlus (1993): Daedalus Manual's. Maintenance Manual AA5000 Multispectral Infrared and Visibile Imaging Spectrometer (MIVIS), vol. 2.

LECHI, G., (2000): Calibrazione radiometrica su 102 canali distribuiti sulle bande dal visibile all'infrarosso termico dello scanner aerotrasportato MIVIS del CNR, Rapporto Interno CNR IIA.

Milton, E.J., D.R. EMERY and C.H. KerR (1997): NERC equipment pool for field spectroscopy. The 21 st century observations and interactions, in Proceedings of the 23rd Annual Conference of the Remote Sensing Society, Nottingham, U.K., 159-164.

RolLIN, E.M. (2000): Monitoring the average spectral radiance of the Daedalus AB532A calibration bench for Daedalus 1268 ATM scanner, in Activities of the NERC Equipment Pool for Field Spectroscopy (EPFS) in support of the NERC Airborne Remote Sensing Facility, British Geological Survey, University of Southampton, 33-43.

SLATER, P.N. (1980): Remote Sensing - Optics and Optical Systems (Addison-Wesley Publishing Company, Reading, Massachusetts, U.S.A.), pp. 575.

TANRÉ, D., B.N. Holben and Y.J. KaufMan (1992): Atmospheric correction algorithm for NOAA-AVHRR Products: theory and application, IEEE Trans. Geosci. Remote Sensing, 30, 231-248.

Vermote, E.F., N. El Saleous, C.O. Justice, Y.J. Kaufman, J.L. Privette, L.A. Remer, J.C. Roger and D. TANRÉ (1997): Atmospheric correction of visible to middle-infrared EOS-MODIS data over land surfaces: Background, operational algorithm and validation, $J$. Geophys. Res., 102 (D14), 17131-17141. 